\title{
TRANSFORMATION DE LA FAUNE DES POISSONS DANS LA RIVIERE LOT
}

\author{
par J. N. TOURENQ ${ }^{1}$ et F. DAUBA ${ }^{2}$
}

Les perturbations provoquées par la construction de grands réservoirs sur le Haut Lot et la Truyère ont modifié la répartition des principales espèces de poissons, notamment dans la zone à barbeaux. Cependant, le schéma piscicole de la rivière reste classique et, pour l'aval, caractéristique d'une rivière de plaine très poissonneuse.

\section{Changes in the species composition of fish in the River Lot.}

The disturbances in the river caused by the construction of large reservoirs in the upper Lot and its tributary the Truyere have modified the distribution of the principal fish species, particularly in the barbel zone.

However the overall distribution of fish species in the river remains normal and downstream the distribution is characteristic of a lowland river with abundant fish.

L'objet de cette note est de dresser un inventaire des poissons du Lot tout en essayant de comparer la distribution actuelle des espèces à celle qui existait avant la construction des grands barrages amont.

Les résultats présentés proviennent d'enquêtes effectuées auprès des riverains, des pêcheurs et des associations de pêche ainsi que des captures réalisées ces dernières années en divers points de la rivière : pêches au filet et électrique notamment à l'occasion d'études localisées (Labat et al., 1977).

\section{RESULTATS}

L'inventaire des poissons du Lot s'établit comme suit :

- carnassiers : truite Salmo trutta f. fario ; brochet Esox lucius (L.) ; black bass Micropterus salmoïdes (Lacépède); sandre Lucioperca lucioperca (L.); perche Perca fluviatilis (L.) ; perche soleil Lepo-

1. Laboratoire d'Hydrobiologie (ERA 702), Université Paul-Sabatier, 118, route de Narbonne, 31077 Toulouse Cedex.

2. Laboratoire d'Ichtyologie appliquée, ENSAT, 145, avenue de Muret, 31076 Toulouse Cedex. 
TableaU I. - Distribution des poissons dans le Lot.

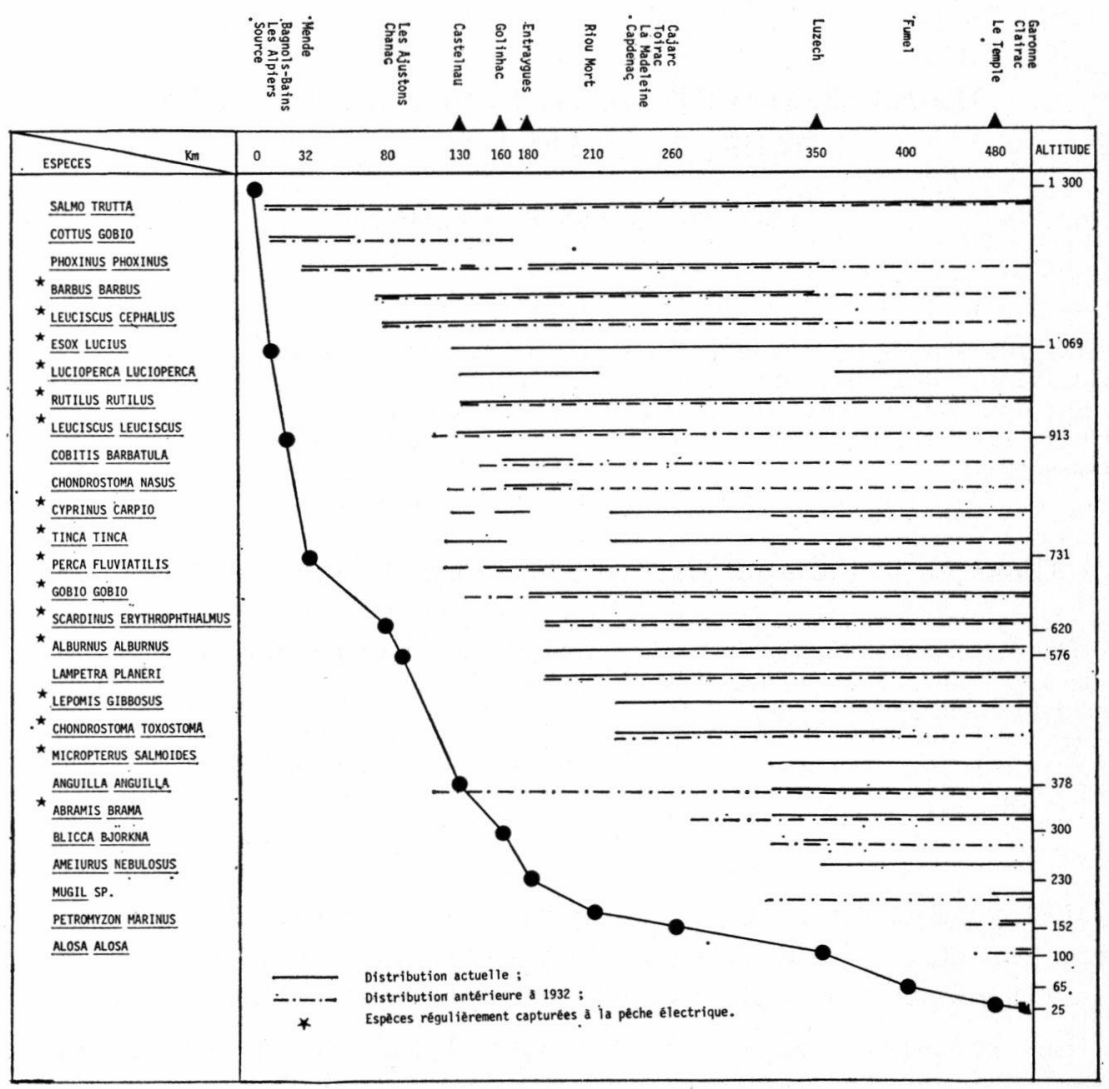

mis gibbosus (L.) ; anguille Anguilla anguilla (L.) ; chabot Cottus gobio (L.).

- omnivores: poisson-chat Ameiurus nebulosus (Le Sueur); ablette Alburnus alburnus (L.) ; chevesne Leuciscus cephalus (L.) ; gardon Rutilus rutilus (L.) ; rotengle Scardinius erythrophthalmus (L.) ; brême Abramis brama (L.) ; brême bordelière Blicca björkna (L.).

- fond : goujon Gobio gobio (L.) ; vandoise Leuciscus leuciscus (L.) ; barbeau Barbus barbus (L.) ; vairon Phoxinus phoxinus (L.) ; loche franche Cobitis barbatula (L.); hotu Chondrostoma nasus (L.).

- fouisseurs : carpe Cyprinus carpio (L.) ; tanche Tinca tinca (L.) ; soffie Chondrostoma toxostoma Vallot.

- migrateurs : mulet Mugil sp.; lamproie marine Petromyzon marinus (L.) ; lamproie de Planer Lampetra planeri (Bloch) ; alose Alosa alosa (L.) ; saumon Salmo salar (L.) (abondant jusqu'au milieu du XVIII $^{\mathrm{e}}$ siècle) actuellement disparu. 
- autre : lamproie de Planer Lampetra planeri (Bloch).

Le tableau I rend compte de la distribution des espèces d'amont en aval sur le Lot. Comme on peut le noter sur ce tableau, la construction à partir de 1932 des grands barrages sur le Haut Lot et la Truyère, ainsi que la construction des barrages au fil de l'eau en moyenne et basse vallée, ont transformé la distribution des poissons dans le Lot. A Entraygues, l'abaissement de la température de l'eau a provoqué la diminution rapide des poissons blancs à l'exception du chevesne, la truite devenant à ce niveau l'espèce dominante (rôle autrefois tenu par la vandoise). Malgré de nombreux alevinages, le peuplement d'origine n'a jamais pu être reconstitué. Plusieurs espèces ont été introduites dans ces grands réservoirs : carpe, tanche, gardon, brochet, sandre. Cette dernière espèce prolifère actuellement au détriment de la truite. La construction de barrages au fil de l'eau, notamment à Luzech, a arrêté la remontée des anguilles et des mulets, de même que l'exten-

Tableau II. - Evolution du peuplement dans le réservoir du Temple (km 480) depuis la construction du barrage en 1948.

\begin{tabular}{|c|c|}
\hline Espèces & Observations \\
\hline Salmo trutta & $\begin{array}{l}\text { disparu du réservoir mais toujours présent dans } \\
\text { les petits ruisseaux (Lède, Tolzac). }\end{array}$ \\
\hline Esox lucius & introduit en 1948, stable. \\
\hline Perca fluviatilis & stable. \\
\hline Lepomis gibbosus & nette diminution. \\
\hline Micropterus salmoïdes & introduit en 1948, diminue. \\
\hline Anguilla anguilla & nette diminution. \\
\hline Lucioperca lucioperca & introduit en 1948 , en augmentation. \\
\hline Abramis brama & augmentation. \\
\hline Rutilus rutilus & augmentation. \\
\hline Scardinius erythrophthalmus & stable. \\
\hline Alburnus alburnus & diminution. \\
\hline Leuciscus cephalus & disparu. \\
\hline Ameiurus nebulosus & très nette prolifération. \\
\hline Leuciscus leuciscus & disparu. \\
\hline Gobio gobio & disparu. \\
\hline Barbus barbus & disparu. \\
\hline Chondrostoma nasus & disparu. \\
\hline Cyprinus carpio & stable, mais pas de carpillons. \\
\hline Tinca tinca & en augmentation. \\
\hline Chondrostoma toxostoma & disparu. \\
\hline Mugil sp. & disparu. \\
\hline Petromyzon marinus & disparu - présent en aval du barrage. \\
\hline Alosa alosa & disparu. \\
\hline Lampetra planeri & stable. \\
\hline
\end{tabular}


Tableau III. - Evolution du peuplement dans le réservoir de Luzech ( $\mathrm{km} \mathrm{350)}$ depuis la construction du barrage en 1950.

\begin{tabular}{ll}
\hline \multicolumn{1}{c}{ Espèces } & \multicolumn{1}{c}{ Observations } \\
\cline { 1 - 2 } Salmo trutta & $\begin{array}{l}\text { disparu en 1950, mais présent dans les petits ruis- } \\
\text { seaux. }\end{array}$ \\
* Esox lucius & introduit en 1945, stable. \\
* Perca fluviatilis & uniquement petite taille. \\
* Lepomis gibbosus & nanisme. \\
* Micropterus salmoïdes & introduit en 1945, stable. \\
Anguilla anguilla & disparu, réintroduit en 1976. \\
* Abramis brama & légère diminution. \\
Blicca björkna & légère diminution. \\
* Rutilus rutilus & introduit en 1924, en nette diminution. \\
* Scardinius erythrophthalmus & introduit en 1924, en nette diminution. \\
* Alburnus alburnus & introduit en 1945, en nette augmentation. \\
Leuciscus cephalus & en diminution. \\
Leuciscus leuciscus & disparu en 1950. \\
Gobio gobio & nette diminution. \\
Barbus barbus & très rare. \\
Phoxinus phoxinus & disparu en 1950. \\
Cyprinus carpio & stable. \\
* Tinca tinca & introduit en 1924, stable. \\
Chondrostoma toxostoma & stable. \\
Mugil sp. & disparu. \\
\hline &
\end{tabular}

* régulièrement capturés à la pêche électrique.

sion vers l'amont du poisson-chat. En 1976, des civelles ont été introduites en amont de Luzech.

Les tableaux II, III, IV et V résument l'évolution du peuplement dans les réservoirs du Temple au $\mathrm{km} 480$ (barrage construit en 1948), de Luzech : km 350 (barrage construit en 1950), au confluent LotTruyère : km 180, dans les barrages de Golinhac: km 160 (1960) et Castelnau : km 130 (1951). 
Tableau IV. - Evolution du peuplement au confluent Lot-Truyère ( $k m$ 230) au cours des dernières années.

\begin{tabular}{|c|c|}
\hline Espèces & Observations \\
\hline Salmo trutta & prolifération après 1932. \\
\hline Esox lucius & $\begin{array}{l}\text { présent en petite quantité depuis quelques an- } \\
\text { nées (5). }\end{array}$ \\
\hline Anguilla anguilla & nette diminution, rare. \\
\hline Lucioperca lucioperca & introduit depuis 5 ans, nette augmentation. \\
\hline Rutilus rutilus & $\begin{array}{l}\text { récemment introduit, alevinages réguliers, } \\
\text { pas de reproduction. }\end{array}$ \\
\hline Scardinius erythrophthalmus & diminution après 1932, actuellement stable. \\
\hline Leuciscus cephalus & stable. \\
\hline Leuciscus leuciscus & diminution après 1932, actuellement stable. \\
\hline Gobio gobio & nette diminution. \\
\hline Barbus barbus & nette diminution. \\
\hline Chondrostoma nasus & nette diminution. \\
\hline Phoxinus phoxinus & stable. \\
\hline Cobitis barbatula & stable. \\
\hline Cyprinus carpio & $\begin{array}{l}\text { récemment introduit, alevinages réguliers, pas de } \\
\text { reproduction. }\end{array}$ \\
\hline Tinca tinca & $\begin{array}{l}\text { récemment introduit, alevinages réguliers, pas de } \\
\text { reproduction. }\end{array}$ \\
\hline Lampetra planeri & stable. \\
\hline
\end{tabular}

Tableau V. - Evolution du peuplement dans la région d'Estaing et d'Espalion. Barrages de Golinhac (km 160), année de construction 1960 et de Castelnau (km 130), année de construction 1951.

\begin{tabular}{ll}
\hline \multicolumn{1}{c}{ Espèces } & \multicolumn{1}{c}{ Observations } \\
\hline Salmo trutta & population stable. \\
Esox lucius & introduction récente, stable. \\
Perca fluviatilis & introduction récente, stable. \\
Anguilla anguilla & disparu. \\
Lucioperca lucioperca & introduction récente, en nette augmentation. \\
Rutilus rutilus & introduction récente, stable. \\
Leuciscus cephalus & stable. \\
Leuciscus leuciscus & légère diminution. \\
Gobio gobio & disparu. \\
Barbus barbus & stable. \\
Phoxinus phoxinus & disparu. \\
Chondrostoma nasus & disparu. \\
Cyprinus carpio & introduction récente, stable. \\
Tinca tinca & introduction récente, stable. \\
\hline
\end{tabular}




\section{CONCLUSION}

Le Lot comprend donc une faune de poissons classique pour une rivière de plaine. La région salmonicole (Huet, 1949) y est réduite à une trentaine de kilomètres.

La construction depuis 1932 des grands barrages réservoirs en amont a eu pour effet :

1) de perturber la zone à barbeaux et,

2) de diminuer la température moyenne des eaux à l'aval des réservoirs, dans la zone située en amont du Lot canalisé.

Ce deuxième effet a limité l'extension de la zone à brême vers l'amont, malgré la multiplication des barrages au fil de l'eau. Enfin, les deux derniers réservoirs de l'aval (Le Temple et Villeneuve) demeurent les plus poissonneux.

\section{TRAVAUX CITES}

Labat (R.), Roqueplo (C.), Ricard (J.-M.), Lim (P.) et Burgat (M.). 1977. - Actions écotoxicologiques de certains métaux $(\mathrm{Cu}, \mathrm{Zn}, \mathrm{Pb}, \mathrm{Cd})$ chez les poissons dulçaquicoles de la rivière Lot. Annls Limnot., 13 (2): 191-207.

HuET (M.). 1949. - Aperçu des relations entre la pente et les populations piscicoles dans les eaux courantes. Rev. suisse Hydrol., 11 (3-4) : 332-351. 